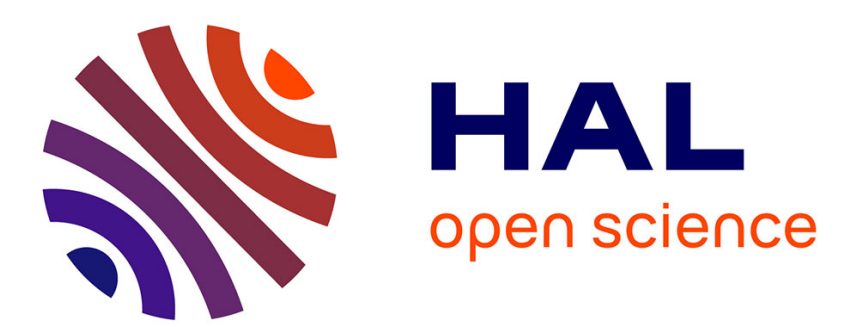

\title{
A summary of numerical methods for time-dependent advection-dominated partial differential equations
}

\author{
Richard E. Ewing, Hong Wang
}

\section{To cite this version:}

Richard E. Ewing, Hong Wang. A summary of numerical methods for time-dependent advectiondominated partial differential equations. Journal of Computational and Applied Mathematics, 2001, 128 (1-2), pp.423-445. 10.1016/S0377-0427(00)00522-7 . hal-01635217

\section{HAL Id: hal-01635217 https://hal.science/hal-01635217}

Submitted on 14 Nov 2017

HAL is a multi-disciplinary open access archive for the deposit and dissemination of scientific research documents, whether they are published or not. The documents may come from teaching and research institutions in France or abroad, or from public or private research centers.
L'archive ouverte pluridisciplinaire HAL, est destinée au dépôt et à la diffusion de documents scientifiques de niveau recherche, publiés ou non, émanant des établissements d'enseignement et de recherche français ou étrangers, des laboratoires publics ou privés. 


\title{
A summary of numerical methods for time-dependent advection-dominated partial differential equations
}

\author{
Richard E. Ewing ${ }^{\mathrm{a}, *}$, Hong Wang ${ }^{\mathrm{b}}$ \\ ${ }^{a}$ Institute for Scientific Computation, Texas A\&M University, College Station, Texas 77843-3404, USA \\ ${ }^{\mathrm{b}}$ Department of Mathematics, University of South Carolina, Columbia, South Carolina 29208, USA
}

We give a brief summary of numerical methods for time-dependent advection-dominated partial differential equations (PDEs), including first-order hyperbolic PDEs and nonstationary advection-diffusion PDEs. Mathematical models arising in porous medium fluid flow are presented to motivate these equations. It is understood that these PDEs also arise in many other important fields and that the numerical methods reviewed apply to general advection-dominated PDEs. We conduct a brief historical review of classical numerical methods, and a survey of the recent developments on the Eulerian and characteristic methods for time-dependent advection-dominated PDEs. The survey is not comprehensive due to the limitation of its length, and a large portion of the paper covers characteristic or Eulerian-Lagrangian methods.

Keywords: Advection-diffusion equations; Characteristic methods; Eulerian methods; Numerical simulations

\section{Mathematical models}

We present mathematical models arising in subsurface porous medium fluid flow (e.g. subsurface contaminant transport, reservoir simulation) to motivate time-dependent advection-dominated PDEs. These types of PDEs also arise in many other important fields, such as the mathematical modeling of aerodynamics, fluid dynamics (e.g. Euler equations, Navier-Stokes equations) [70,93], meteorology [90], and semiconductor devices [72].

\footnotetext{
${ }^{*}$ Corresponding author.

E-mail address: richard-ewing@tamu.edu (R.E. Ewing).
} 


\subsection{Miscible flows}

A mathematical model used for describing fully saturated fluid flow processes through porous media is derived by using the mass balance equation for the fluid mixture $[5,40]$

$$
\frac{\partial}{\partial t}(\phi \rho)-\nabla \cdot\left(\frac{\rho \boldsymbol{K}}{\mu}(\nabla p-\rho \boldsymbol{g})\right)=q, \quad \boldsymbol{x} \in \Omega, t \in[0, T] .
$$

Here $\Omega$ is the physical domain, $\boldsymbol{u}, p$, and $\rho$ are the Darcy velocity, the pressure, and the mass density of the fluid, $\boldsymbol{K}(\boldsymbol{x})$ is the absolute permeability of the medium, $\mu$ is the dynamic viscosity of the fluid, $\boldsymbol{g}$ is the acceleration vector due to gravity, and $q$ represents the source and sink terms, which is often modeled via point or line sources and sinks.

The transport of a specific component in the fluid mixture is governed by the mass conservation for the component and is expressed as

$$
\frac{\partial(\phi c)}{\partial t}+\nabla \cdot(\boldsymbol{u} c)-\nabla \cdot(\boldsymbol{D}(\boldsymbol{u}) \nabla c)=\bar{c} q, \quad \boldsymbol{x} \in \Omega, t \in[0, T] .
$$

Here $c$, a fraction between 0 and 1 , represents the concentration of the component, $\phi$ is the porosity of the medium, $\bar{c}(\boldsymbol{x}, t)$ is either the specified concentrations of the injected fluids at sources or the resident concentrations at sinks, and $\boldsymbol{D}(\boldsymbol{u})$ is the diffusion-dispersion tensor.

\subsection{Multiphase flows}

When either air or a nonaqueous-phase liquid (NAPL) contaminant is present in groundwater transport processes, this phase is immiscible with the water phase and the two phases flow simultaneously in the flow process. Likewise, in the immiscible displacement in petroleum production, the oil phase and the water phase are immiscible. In both cases, there is no mass transfer between the two phases and so the following equations hold for each phase $[5,17,19,40]$ :

$$
\frac{\partial}{\partial t}\left(\phi \rho_{j} S_{j}\right)-\nabla \cdot\left(\frac{\rho_{j} \boldsymbol{K} k_{r j}}{\mu_{j}} \nabla p_{j}\right)=\rho_{j} q_{j}, \quad \boldsymbol{x} \in \Omega, t \in[0, T] .
$$

Here $S_{j}, \boldsymbol{u}_{j}, \rho_{j}, p_{j}, k_{r j}, \mu_{j}$, and $q_{j}$ are the saturation, velocity, density, pressure, relative permeability, viscosity, and source and sink terms for the phase $j$. The indices $j=\mathrm{n}$ and w stand for the nonwetting and wetting phases, respectively. The saturations $S_{\mathrm{n}}$ and $S_{\mathrm{w}}$ satisfy the relation $S_{\mathrm{n}}+S_{\mathrm{w}}=1$.

Eqs. (1.3) may be rearranged in a form that resembles Eqs. (1.1) and (1.2) by letting $S_{\mathrm{n}}=1-S_{\mathrm{w}}$. The pressure between the two phases is described by the capillary pressure $p_{\mathrm{c}}\left(S_{\mathrm{w}}\right)=p_{\mathrm{n}}-p_{\mathrm{w}}$. The global pressure $p$ and total velocity $\boldsymbol{u}$ of a two-phase flow model is given by the following equations [21]:

$$
S_{\mathrm{n}} \mathrm{C}_{\mathrm{n}} \frac{D p}{D t}-\nabla \cdot(\boldsymbol{K} \lambda \nabla p)=q\left(\boldsymbol{x}, S_{\mathrm{w}}, p\right), \quad \boldsymbol{x} \in \Omega, t \in[0, T],
$$

where $(D / D t)=\phi(\partial / \partial t)+\left(\boldsymbol{u}_{\mathrm{n}} / S_{\mathrm{n}}\right) \cdot \nabla$ and $p=\frac{1}{2}\left(p_{\mathrm{n}}+p_{\mathrm{w}}\right)+\frac{1}{2} \int_{S_{\mathrm{c}}}^{S}\left(\left(\lambda_{\mathrm{n}}-\lambda_{\mathrm{w}}\right) / \lambda\right)\left(\mathrm{d} p_{\mathrm{c}} / \mathrm{d} \xi\right) \mathrm{d} \xi$ with $p_{\mathrm{c}}\left(S_{\mathrm{c}}\right)=0$. The total mobility $\lambda=\lambda_{\mathrm{n}}+\lambda_{\mathrm{w}}$, the phase mobility $\lambda_{j}=k_{r j} / \mu_{j}$, and the compressibility $\mathrm{C}_{j}=\left(1 / \rho_{j}\right)\left(\mathrm{d} \rho_{j} / \mathrm{d} p_{j}\right)$ are functions of time and space. 
The governing equation for the wetting phase now has a form

$$
\phi \frac{\partial S_{\mathrm{w}}}{\partial t}+\nabla \cdot\left(f\left(S_{\mathrm{w}}\right) \boldsymbol{u}-\boldsymbol{D}\left(S_{\mathrm{w}}\right) \nabla S_{\mathrm{w}}\right)=q_{\mathrm{w}}, \quad \boldsymbol{x} \in \Omega, t \in[0, T],
$$

where $\rho_{\mathrm{c}}=\rho_{\mathrm{n}}-\rho_{\mathrm{w}}$. The capillary diffusion $\boldsymbol{D}\left(S_{\mathrm{w}}\right)=-\boldsymbol{K} \lambda_{\mathrm{n}} f_{\mathrm{w}}\left(\mathrm{d} p_{\mathrm{c}} / \mathrm{d} S_{\mathrm{w}}\right)$ and the fractional flow functions $f_{j}=\lambda_{j} / \lambda$.

In practice, the diffusion term in Eq. (1.2) or (1.5) is often a small phenomenon relative to advection. Hence, these equations are time-dependent advection-diffusion partial differential equations (PDEs) in terms of the concentration $c$ or the saturation $S$. In particular, Eq. (1.5) has an $S$-shaped nonlinear flux function $f$ and a degenerate capillary diffusion term $[19,40]$. Sometimes the diffusion phenomenon is so small that its effect is neglected. In this case, Eq. (1.2) or (1.5) is reduced to a first-order hyperbolic PDE. Finally, initial and boundary conditions also need to be specified to close the system $(1.1)-(1.2)$ or $(1.4)-(1.5)$.

\section{Conventional finite difference and finite element methods}

We carry out a brief historical review of classical numerical methods in this section and a survey of the recent developments on the Eulerian and characteristic methods in the next section primarily for time-dependent advection-dominated PDEs, including first-order hyperbolic PDEs and nonstationary advection-diffusion PDEs. Because of the extensive research carried out in these areas, it is impossible to describe adequately all these developments in the space available. Hence, this review is not comprehensive in that we try to describe and review only some representatives of the huge amount of works in the literature. Notice that since relatively more references and survey papers can be found on the Eulerian methods for unsteady state advection-dominated PDEs, we intend to use a relatively large portion to cover characteristic or Eulerian-Lagrangian methods for advection-dominated PDEs. Finally, we refer interested readers to the works of Morton [73] and Roos et al. [86] for detailed descriptions on the recent developments for the numerical methods for stationary advection-diffusion PDEs.

It is well known that advection-dominated PDEs present serious numerical difficulties due to the moving steep fronts present in the solutions of advection-diffusion transport PDEs or shock discontinuities in the solutions of pure advection PDEs or advection-diffusion PDEs with degenerate diffusion. Additional difficulties include the strong couplings and nonlinearities of advection-dominated PDE systems, the effect of the singularities at point/line sources and sinks, the strong heterogeneity of the coefficients, anisotropic diffusion-dispersion in tensor form, and the enormous sizes of field-scale applications.

\subsection{Finite difference methods (FDMs)}

Due to their simplicity, FDMs were first used in solving advection-dominated PDEs. For convenience, of presentation, we consider the one-dimensional constant-coefficient analogue of Eq. (1.2)

$$
\frac{\partial c}{\partial t}+V \frac{\partial c}{\partial x}-D \frac{\partial^{2} c}{\partial x^{2}}=0, \quad x \in(a, b), t \in[0, T]
$$


and assume a uniform spatial and temporal partition $x_{i}=a+i \Delta x$ for $i=0,1, \ldots, I$ with $\Delta x=(b-a) / I$ and $t^{m}=m \Delta t$ for $m=0,1, \ldots, M$ with $\Delta t=T / M$.

We define the Courant number $\mathrm{Cr}=V \Delta t / \Delta x$ and the Peclet number $\mathrm{Pe}=V \Delta x / D$. It is known that the solution to the space-centered explicit scheme

$$
\frac{c_{i}^{m+1}-c_{i}^{m}}{\Delta t}+V \frac{c_{i+1}^{m}-c_{i-1}^{m}}{2 \Delta x}-D \frac{c_{i+1}^{m}-2 c_{i}^{m}+c_{i-1}^{m}}{(\Delta x)^{2}}=0
$$

does not oscillate only when the Peclet number $\mathrm{Pe} \leqslant 2$ and the CFL condition $(\mathrm{Cr} \leqslant 1)$ is satisfied $[30,81]$. For $\mathrm{Pe}>2$, damped oscillations occur with nonreal eigenvalues [48,81]. Furthermore, for the linear hyperbolic PDE

$$
\frac{\partial c}{\partial t}+V \frac{\partial c}{\partial x}=0, \quad x \in(a, b), t \in[0, T]
$$

which can be viewed as a limiting case of $D \rightarrow 0$ in Eq. (2.1), the corresponding scheme to scheme (2.2)

$$
\frac{c_{i}^{m+1}-c_{i}^{m}}{\Delta t}+V \frac{c_{i+1}^{m}-c_{i-1}^{m}}{2 \Delta x}=0
$$

is unconditionally unstable $[48,94]$.

The upwind FDM (UFDM) uses a one-sided finite difference in the upstream direction to approximate the advection term in the transport PDE (2.1) and can be expressed as follows (assuming $V>0)$ :

$$
\frac{c_{i}^{m+1}-c_{i}^{m}}{\Delta t}+V \frac{c_{i}^{m}-c_{i-1}^{m}}{2 \Delta x}-D \frac{c_{i+1}^{m}-2 c_{i}^{m}+c_{i-1}^{m}}{(\Delta x)^{2}}=0 .
$$

The Lax-Friedrichs scheme

$$
\frac{c_{i}^{m+1}-\left(c_{i+1}^{m}+c_{i-1}^{m}\right) / 2}{\Delta t}+V \frac{c_{i+1}^{m}-c_{i-1}^{m}}{2 \Delta x}-D \frac{c_{i+1}^{m}-2 c_{i}^{m}+c_{i-1}^{m}}{(\Delta x)^{2}}=0
$$

is obtained by replacing $c_{i}^{m}$ in the first term in Eq. (2.2) by its mean value $\left(c_{i+1}^{m}+c_{i-1}^{m}\right) / 2$.

Remark 1. Schemes (2.5) and (2.6) eliminate the nonphysical oscillations present in Scheme (2.2), and generate stable solutions even for very complicated multiphase and multicomponent flows. It can be shown that the UFDM scheme is actually a second-order approximation to Eq. (2.1) with a modified diffusion $D(1+(\mathrm{Pe} / 2)(1-\mathrm{Cr}))$, while the Lax-Friedrichs scheme is a second-order approximation to Eq. (2.1) with an extra numerical diffusion $\left((\Delta x)^{2} / 2 \Delta t\right)\left(1-\mathrm{Cr}^{2}\right)[40,59,70]$. Hence, these methods introduce excessive numerical diffusion and the numerical solutions are dependent upon grid orientation. Detailed description on the theory and the use of modified equations can be found in $[59,70,110]$.

The Lax-Wendroff scheme is based on the Taylor series expansion and Eq. (2.3)

$$
\begin{aligned}
c\left(x, t^{m+1}\right) & =c\left(x, t^{m}\right)+\Delta t \frac{\partial c\left(x, t^{m}\right)}{\partial t}+\frac{(\Delta t)^{2}}{2} \frac{\partial^{2} c\left(x, t^{m}\right)}{\partial t^{2}}+\mathrm{O}\left((\Delta t)^{3}\right) \\
& =c\left(x, t^{m}\right)-V \Delta t \frac{\partial c\left(x, t^{m}\right)}{\partial x}+\frac{(V \Delta t)^{2}}{2} \frac{\partial^{2} c\left(x, t^{m}\right)}{\partial x^{2}}+\mathrm{O}\left((\Delta t)^{3}\right) .
\end{aligned}
$$


Dropping the $\mathrm{O}\left((\Delta t)^{3}\right)$ term in Eq. (2.7) and using centered differences to approximate the spatial derivatives yields the Lax-Wendroff scheme

$$
c_{i}^{m+1}=c_{i}^{m}-\frac{\mathrm{Cr}}{2}\left(c_{i+1}^{m}-c_{i-1}^{m}\right)+\frac{\mathrm{Cr}^{2}}{2}\left(c_{i+1}^{m}-2 c_{i}^{m}+c_{i-1}^{m}\right),
$$

which is a second-order scheme.

The Beam-Warming scheme is a one-sided version of the Lax-Wendroff scheme. It uses secondorder accurate one-sided differences to approximate the spatial derivatives in Eq. (2.7)

$$
c_{i}^{m+1}=c_{i}^{m}-\frac{\mathrm{Cr}}{2}\left(3 c_{i}^{m}-4 c_{i-1}^{m}+c_{i-2}^{m}\right)+\frac{\mathrm{Cr}^{2}}{2}\left(c_{i}^{m}-2 c_{i-1}^{m}+c_{i-2}^{m}\right) .
$$

Remark 2. The Lax-Wendroff scheme and the Beam-Warming scheme give third-order approximations to the modified advection-dispersion equation

$$
\frac{\partial c}{\partial t}+V \frac{\partial c}{\partial x}-\beta \frac{\partial^{3} c}{\partial x^{3}}=0, \quad x \in(a, b), t \in[0, T]
$$

with $\beta=\left(V(\Delta x)^{2} / 6\right)\left(\mathrm{Cr}^{2}-1\right)$ for $(2.8)$ and $\left(V(\Delta x)^{2} / 6\right)\left(2-3 \mathrm{Cr}+\mathrm{Cr}^{2}\right)$ for (2.9). The theory of dispersive waves and its utility in the study of numerical methods are covered in [97,112], which show that the Lax-Wendroff scheme tends to develop oscillations behind shock fronts while the Beam-Warming scheme tends to develop oscillations in front of shock fronts.

Remark 3. Solving Eq. (2.3) yields $c\left(x, t^{m+1}\right)=c\left(x-V \Delta t, t^{m}\right)$. When the CFL condition is satisfied, the UPFD or the Lax-Friedrichs scheme can be viewed as an linear interpolation of $c\left(x-V \Delta t, t^{m}\right)$ by the nodal values $c\left(x_{i-1}, t^{m}\right)$ and $c\left(x_{i}, t^{m}\right)$, or $c\left(x_{i-1}, t^{m}\right)$ and $c\left(x_{i+1}, t^{m}\right)$, respectively. This explains why these schemes are free of oscillations and introduce smearing from another point of view [70]. Second, because UFDM takes advantage of upstream information, it is slightly more accurate than the Lax-Friedrichs scheme. On the other hand, the latter is symmetric and can be easily implemented, which is an important feature for nonlinear hyperbolic conservation laws. In contrast, the LaxWendroff scheme (2.8) or Beam-Warming scheme (2.9) can be viewed as a quadratic interpolation of $c\left(x-V \Delta t, t^{m}\right)$ by the nodal values $c\left(x_{i-1}, t^{m}\right), c\left(x_{i}, t^{m}\right)$, and $c\left(x_{i+1}, t^{m}\right)$, or $c\left(x_{i}, t^{m}\right), c\left(x_{i+1}, t^{m}\right)$, and $c\left(x_{i+2}, t^{m}\right)$. This is why they introduce oscillations across shock discontinuities.

The leap-frog scheme for Eq. (2.3) is obtained by replacing the forward difference in time in (2.4) by a centered difference

$$
\frac{c_{i}^{m+1}-c_{i}^{m-1}}{\Delta t}+V \frac{c_{i+1}^{m}-c_{i-1}^{m}}{2 \Delta x}=0 .
$$

Scheme (2.10) has an improved truncation error of $\mathrm{O}\left((\Delta x)^{2}+(\Delta t)^{2}\right)$, but it is a multi-level scheme. This leads to increased computational storage, a particular disadvantage for large multi-dimensional nonlinear systems.

These methods can be extended to solve nonlinear hyperbolic conservation laws

$$
\frac{\partial c}{\partial t}+\frac{\partial f(c)}{\partial x}=0, \quad x \in(a, b), t \in[0, T]
$$


and their viscous analogue [48,70]. For example, a large class of upwind schemes have been developed, based on the Godunov scheme [50]; they have often been presented in terms of Riemann solvers. The Lax-Friedrichs scheme is a basis for the development of nonoscillatory central schemes (see e.g. [75]).

\subsection{Galerkin and Petrov-Galerkin finite element methods (FEMS)}

Many FEM schemes have been developed in parallel. For instance, the Galerkin and PetrovGalerkin FEMs that are analogues to Scheme (2.2) and the UFDM (2.5) for Eq. (2.1) can be uniformly written as follows:

$$
\begin{aligned}
\int_{a}^{b} c\left(x, t^{m+1}\right) w_{i}(x) \mathrm{d} x-\int_{a}^{b} c\left(x, t^{m}\right) w_{i}(x) \mathrm{d} x \\
\quad+\gamma \Delta t\left[\int_{a}^{b} D \frac{\partial c\left(x, t^{m+1}\right)}{\partial x} \frac{\partial w_{i}(x)}{\partial x} \mathrm{~d} x+\int_{a}^{b} V \frac{\partial c\left(x, t^{m+1}\right)}{\partial x} w_{i}(x) \mathrm{d} x\right] \\
=-(1-\gamma) \Delta t\left[\int_{a}^{b} D \frac{\partial c\left(x, t^{m}\right)}{\partial x} \frac{\partial w_{i}(x)}{\partial x} \mathrm{~d} x+\int_{a}^{b} V \frac{\partial c\left(x, t^{m}\right)}{\partial x} w_{i}(x) \mathrm{d} x\right]
\end{aligned}
$$

Here $c\left(x, t^{m+1}\right)$ is a piecewise-linear trial function. In the linear Galerkin FEM, the test functions $w_{i}(x)$ are standard hat functions centered at the node $x_{i}$ and correspond to the space-centered scheme (2.2) (see e.g. [40,48]).

In the quadratic Petrov-Galerkin FEM (QPG), the test functions are constructed by adding an asymmetric perturbation to the original piecewise-linear hat functions $[4,15,22]$

$$
w_{i}(x)= \begin{cases}\frac{x-x_{i-1}}{\Delta x}+v \frac{\left(x-x_{i-1}\right)\left(x_{i}-x\right)}{\Delta x^{2}}, & x \in\left[x_{i-1}, x_{i}\right], \\ \frac{x_{i+1}-x}{\Delta x}-v \frac{\left(x-x_{i}\right)\left(x_{i+1}-x\right)}{\Delta x^{2}}, & x \in\left[x_{i}, x_{i+1}\right], \\ 0 & \text { otherwise. }\end{cases}
$$

With a choice of $v=3$, the QPG reproduces the UFDM. With an optimal choice of $v=3[\operatorname{coth}(\mathrm{Pe} / 2)-$ $2 / \mathrm{Pe}$ ], the QPG is reduced to the optimal FDM of Allen and Southwell [1]. For a stationary analogue of Eq. (2.1), the QPG method yields solutions that coincide with the exact solution at the nodal points, and minimizes the errors in approximating spatial derivatives [4,15]. However, the QPG is susceptible to strong time truncation errors that introduce numerical diffusion and the restrictions on the size of the Courant number, and hence tends to be ineffective for transient advection-dominated PDEs. 
In the cubic Petrov-Galerkin FEM (CPG), the test functions are defined as the original piecewiselinear hat functions with a symmetric cubic perturbation added to each nonzero piece $[10,111]$

$$
w_{i}(x)= \begin{cases}\frac{x-x_{i-1}}{\Delta x}+v \frac{\left(x-x_{i-1}\right)\left(x_{i}-x\right)\left(x_{i-1}+x_{i}-2 x\right)}{\Delta x^{3}}, & x \in\left[x_{i-1}, x_{i}\right], \\ \frac{x_{i+1}-x}{\Delta x}-v \frac{\left(x-x_{i}\right)\left(x_{i+1}-x\right)\left(x_{i}+x_{i+1}-2 x\right)}{\Delta x^{3}}, & x \in\left[x_{i}, x_{i+1}\right], \\ 0 & \text { otherwise. }\end{cases}
$$

Here $v=5 \mathrm{Cr}^{2}$. The CPG intends to use nonzero spatial error to cancel the temporal error to improve the overall accuracy. In these treatments the effects on mass balance come from spatial dependence of test functions in the first terms on both the sides of Eq. (2.12). Detailed descriptions of the FDMs and FEMs that have been used in the petroleum industry can be found in $[40,89]$.

Corresponding to the Lax-Wendroff scheme (2.8) and the leap-frog scheme (2.10) is the TaylorGalerkin scheme

$$
\begin{gathered}
\frac{c_{i+1}^{m+1}-c_{i+1}^{m-1}}{6}+\frac{2\left(c_{i}^{m+1}-c_{i}^{m-1}\right)}{3}+\frac{c_{i-1}^{m+1}-c_{i-1}^{m-1}}{6} \\
=-\frac{\mathrm{Cr}}{2}\left(c_{i+1}^{m}-c_{i-1}^{m}\right)+\frac{\mathrm{Cr}^{2}}{2}\left(c_{i+1}^{m}-2 c_{i}^{m}+c_{i-1}^{m}\right),
\end{gathered}
$$

and the leap-frog Galerkin scheme

$$
\frac{c_{i+1}^{m+1}-c_{i+1}^{m-1}}{6 \Delta t}+\frac{2\left(c_{i}^{m+1}-c_{i}^{m-1}\right)}{3 \Delta t}+\frac{c_{i-1}^{m+1}-c_{i-1}^{m-1}}{6 \Delta t}+V \frac{c_{i+1}^{m}-c_{i-1}^{m}}{2 \Delta x}=0 .
$$

In addition, a wide variety of other methods can be devised for advection-dominated transport PDEs by using different FDM and FEM approximations, or Taylor expansions. Many large-scale simulators use fully implicit discretization so that large time steps can be allowed. However, in implicit methods, the temporal error and the spatial error add together. Hence, increasing the size of time steps can significantly reduce the accuracy of the solutions [40]. This is also observed computationally [106]. In contrast, in explicit schemes the temporal error and the spatial error cancel each other. Hence, reducing the time step size further with fixed spatial step size will actually reduce the accuracy of the numerical solutions. The sizes of spatial grids and temporal steps have to be reduced simultaneously to improve the accuracy of the solutions, leading to significantly increased overall computational and storage cost [106].

\section{Recent developments for advection-diffusion PDEs}

Recent developments in effectively solving advection-diffusion PDEs have generally been along one of two approaches: Eulerian or characteristic Lagrangian methods. Eulerian methods use the standard temporal discretization, while the main distinguishing feature of characteristic methods is the use of characteristics to carry out the discretization in time. 


\subsection{Eulerian methods for advection-diffusion PDEs}

Many methods directly apply to a nonconservative analogue of Eq. (1.2)

$$
\phi \frac{\partial c}{\partial t}+\boldsymbol{u} \cdot \nabla c-\nabla \cdot(\boldsymbol{D} \nabla c)=\bar{c} q, \quad \boldsymbol{x} \in \Omega, t \in[0, T] .
$$

\subsubsection{The streamline diffusion finite element method (SDFEM)}

The SDFEM directly applies to Eq. (3.1). It is based on the framework of space-time FEMs on the space-time strip $\bar{\Omega} \times\left[t^{m}, t^{m+1}\right]$, and uses continuous and piecewise polynomial trial and test functions in space as standard FEM but a discontinuous Galerkin approximation in time at time level $t^{m}$ and $t^{m+1}$ such that

$$
\begin{aligned}
\int_{t^{m}}^{t^{m+1}} & \int_{\Omega}\left[\phi \frac{\partial c}{\partial t}+\boldsymbol{u} \cdot \nabla c-\nabla \cdot(\boldsymbol{D} \nabla c)\right]\left[w+\delta\left(\phi \frac{\partial w}{\partial t}+\boldsymbol{u} \cdot \nabla w\right)\right] \mathrm{d} \boldsymbol{x} \mathrm{d} t \\
& +\int_{t^{m}}^{t^{m+1}} \int_{\Omega} \nabla w \cdot(\boldsymbol{D} \nabla c) \mathrm{d} \boldsymbol{x} \mathrm{d} t+\int_{\Omega} c\left(\boldsymbol{x}, t_{+}^{m}\right) w\left(\boldsymbol{x}, t_{+}^{m}\right) \mathrm{d} \boldsymbol{x} \\
= & \int_{t^{m}}^{t^{m+1}} \int_{\Omega} \bar{c} q\left[w+\delta\left(\phi \frac{\partial w}{\partial t}+\boldsymbol{u} \cdot \nabla w\right)\right] \mathrm{d} \boldsymbol{x} \mathrm{d} t+\int_{\Omega} c\left(\boldsymbol{x}, t_{-}^{m}\right) w\left(\boldsymbol{x}, t_{+}^{m}\right) \mathrm{d} \boldsymbol{x} .
\end{aligned}
$$

Here $w\left(\boldsymbol{x}, t_{+}^{m}\right)=\lim _{t \rightarrow t^{m}, t>t^{m}} w(\boldsymbol{x}, t)$ and $w\left(\boldsymbol{x}, t_{-}^{m}\right)=\lim _{t \rightarrow t^{m}, t<t^{m}} w(\boldsymbol{x}, t)$. At the initial time step, $c\left(\boldsymbol{x}, t_{-}^{0}\right)=$ $c_{0}(\boldsymbol{x})$ is the prescribed initial condition. The second term on the left-hand side is carried out elementwise, since it is not well defined for continuous and piecewise polynomials. The parameter $\delta$, which determines the amount of numerical diffusion introduced, is typically chosen to be of order $\mathrm{O}\left(\sqrt{(\Delta x)^{2}+(\Delta t)^{2}}\right)$.

The SDFEM was first proposed by Hughes and Brooks [62]. Since then, various SDFEM schemes have been developed and studied extensively by Brooks and Hughes and Hughes [11,61] and Johnson et al. $[52,65]$. The SDFEM adds a numerical diffusion only in the direction of streamlines to suppress the oscillation and does not introduce any crosswind diffusion. However, the undetermined parameter $\delta$ in the SDFEM scheme needs to be chosen very carefully in order to obtain accurate numerical results. An optimal choice of the parameter is heavily problem-dependent. We refer readers to the work of Shih and Elman on the study of the choice $\delta$ in the SDFEM formulation and the related numerical experiments [91].

While the SDFEM can capture a jump discontinuity of the exact solution in a thin region, the numerical solution may develop over- and under-shoots about the exact solution within this layer. A modified SDFEM with improved shock-capturing properties was proposed $[63,66]$, which consists of adding a "shock-capturing" term to the diffusion by introducing a "crosswind" control that is close to the steep fronts or "shocks". This modified SDFEM performs much better in terms of catching the steep fronts or the jump discontinuities of the exact solutions. However, the modified SDFEM is a nonlinear scheme and involves another undetermined parameter.

\subsubsection{Total variation diminishing (TVD) methods}

Notice that when oscillations arise, the numerical solutions will have larger total variation. TVD methods are designed to yield well-resolved, nonoscillatory shock discontinuities by enforcing that 
the numerical schemes generate solutions with nonincreasing total variations. One approach is to take a high-order method and add an additional numerical diffusion term to it. Since this numerical diffusion is needed only near discontinuities, one wants it to vanish sufficiently quickly so that the order of accuracy of the method on smooth regions of the solutions is retained. Hence, the numerical diffusion should depend on the behavior of the solutions, being larger near shock regions than in smooth regions. This leads to a nonlinear method even for the linear advection equation (2.3). The idea of adding a variable amount of numerical diffusion dates back to some of the earliest work on the numerical solution of fluid dynamics $[31,68,100]$. The difficulty with this approach is that it is hard to determine an appropriate amount of numerical diffusion that introduces just enough dissipation without causing excessive smearing.

For this reason, the high-resolution methods developed more recently are based on fairly different approaches, including flux- and slope-limiter approaches that impose the nonoscillatory requirement more directly. In the flux-limiter approach, one first chooses a high-order numerical flux $F_{\mathrm{H}}(c, i)=$ $F_{\mathrm{H}}\left(c_{i-l_{\mathrm{H}}}, c_{i-l+1}, \ldots, c_{i+r_{\mathrm{H}}}\right)$ that generates accurate approximations in smooth regions and a low-order numerical flux $F_{\mathrm{L}}(c, i)=F_{\mathrm{L}}\left(c_{i-l_{\mathrm{L}}}, c_{i-l+1}, \ldots, c_{i+r_{\mathrm{L}}}\right)$ that yields nonoscillatory solutions near shock discontinuities. One then combines $F_{\mathrm{H}}$ and $F_{\mathrm{L}}$ into a single numerical flux $F$, e.g. in the form of

$$
F(c, i)=F_{\mathrm{L}}(c, i)+\Psi(c, i)\left(F_{\mathrm{H}}(c, i)-F_{\mathrm{L}}(c, i)\right),
$$

such that $F$ reduces to $F_{\mathrm{H}}$ in smooth regions and to $F_{\mathrm{L}}$ in shock regions. Here $\Psi(c, i)$, the flux limiter, should be near one in smooth regions and close to zero near shock discontinuities.

The flux-corrected transport (FCT) method of Boris and Book can be viewed as one of the earliest flux limiter methods $[8,9,114]$. In the FCT method, an anti-diffusive term (i.e., the correction term in (3.2)) is added to reduce the excessive numerical diffusion introduced by the lower-order flux $F_{\mathrm{L}}$ as much as possible without increasing the total variation of the solution.

Sweby studied a family of flux-limiter methods in [95]. By choosing

$$
F_{\mathrm{L}}\left(c^{m}, i\right)=V c_{i}^{m} \quad \text { and } \quad F_{\mathrm{H}}\left(c^{m}, i\right)=V c_{i}^{m}+\frac{1}{2} V(1-\mathrm{Cr})\left(c_{i+1}^{m}-c_{i}^{m}\right),
$$

to be the first-order upwind flux in (2.5) and the Lax-Wendroff flux in (2.8) and using (3.2), a family of flux-limiter methods can be defined

$$
c_{i}^{m+1}=c_{i}^{m}-\frac{\Delta t}{\Delta x}\left[F\left(c^{m}, i\right)-F\left(c^{m}, i-1\right)\right]
$$

with the flux $F\left(c^{m}, i\right)$ being given by

$$
F\left(c^{m}, i\right)=V c_{i}^{m}+\frac{\Psi\left(c^{m}, i\right)}{2} V(1-\mathrm{Cr})\left(c_{i+1}^{m}-c_{i}^{m}\right) .
$$

One way to measure the smoothness of the solution is to look at the ratio of consecutive gradients and to define the flux limiter $\Psi$ accordingly

$$
\Psi\left(c^{m}, i\right)=\psi\left(\theta_{i}\right) \quad \text { with } \theta_{i}=\frac{c_{i}^{m}-c_{i-1}^{m}}{c_{i+1}^{m}-c_{i}^{m}} .
$$

Sweby obtained algebraic conditions on the limiter functions that guarantee second-order accuracy and the TVD property of the derived methods [95]. Harten proved a sufficient condition on $\psi$ that can be used to impose constraints on $\psi[53,55]$. Among the different choices of limiters are the "superbee" limiter of Roe [85]

$$
(\theta)=\max \{0, \min \{1,2 \theta\}, \min \{\theta, 2\}\}
$$


and a smoother limiter by van Leer [98]

$$
(\theta)=\frac{|\theta|+\theta}{1+|\theta|} .
$$

The extension of flux limiter methods to nonlinear conservation laws and numerical comparisons can be found in $[29,95,115]$.

Another approach is to use slope limiters. These intend to replace the piecewise-constant representation of the solutions in Godunov's method by more accurate representations, and can be expressed in the following steps for Eq. (2.11):

(i) Given the piecewise-constant cell-average representation $\left\{\bar{c}_{i}^{m}\right\}_{i=-\infty}^{i=+\infty}$ of the solution at time level $t^{m}$, define a (e.g., piecewise-linear) reconstruction at time $t^{m}$ by

$$
\hat{c}\left(x, t^{m}\right)=\bar{c}_{i}^{m}+\sigma_{i}^{m}\left(x-x_{i}\right)
$$

for $x$ on the cell $\left[x_{i-1 / 2}, x_{i+1 / 2}\right]$. Here $\sigma_{i}^{m}$ is a slope on the $i$ th cell that is based on the data $\left\{\bar{c}_{i}^{m}\right\}$.

(ii) Solve Eq. (2.11) with the data $\hat{c}\left(x, t^{m}\right)$ at time $t^{m}$ to obtain the solution $c\left(x, t^{m+1}\right)$ at time $t^{m+1}$. (iii) Compute the cell average $\left\{\bar{c}_{i}^{m+1}\right\}_{i=-\infty}^{i=+\infty}$ of the solution $c\left(x, t^{m+1}\right)$ at time $t^{m+1}$.

Note that the cell average of reconstruction (3.4) is equal to $\bar{c}_{i}^{m}$ on the cell $\left[x_{i-1 / 2}, x_{i+1 / 2}\right]$ for any choice of $\sigma_{i}^{m}$. Since Steps 2 and 3 are also conservative, the methods with slope limiters are conservative. Secondly, the choice of $\sigma_{i}^{m}=0$ in Eq. (3.4) recovers Godunov's method. It is well known that Godunov's method generates solutions with excessive numerical diffusion. More accurate reconstructions, such as Eq. (3.4), could be used to reduce the numerical diffusion and to improve the accuracy of the numerical solutions.

In the context of the linear advection PDE (2.3), the solution of Step (ii) is simply $c\left(x, t^{m+1}\right)=$ $\hat{c}\left(x-V \Delta t, t^{m}\right)$. Computing the cell average of $c\left(x, t^{m+1}\right)$ in Step (iii) leads to the following expression:

$$
\bar{c}_{i}^{m+1}=\bar{c}_{i}^{m}-\operatorname{Cr}\left(\bar{c}_{i}^{m}-\bar{c}_{i-1}^{m}\right)-\frac{\Delta x}{2} \operatorname{Cr}(1-\operatorname{Cr})\left(\sigma_{i}^{m}-\sigma_{i-1}^{m}\right) .
$$

A natural choice of $\sigma_{i}^{m}=\left(\bar{c}_{i+1}^{m}-\bar{c}_{i}^{m}\right) / \Delta x$ in Eq. (3.5) leads to the Lax-Wendroff method. Thus, it is possible to obtain second-order accuracy by this approach. Secondly, the slope-limiter methods could generate oscillatory solutions (since the Lax-Wendroff method could do so), if the slope limiters $\sigma_{i}^{m}$ are not chosen properly. Geometrically, the oscillations are due to a poor choice of slopes, which leads to a piecewise-linear reconstruction $\hat{c}\left(x, t^{m}\right)$ with much larger total variation than the given data $\left\{\bar{c}_{i}^{m}\right\}_{i=-\infty}^{i=+\infty}[70]$.

Hence, because of their importance, extensive research has been conducted on how to choose the slope $\sigma_{i}^{m}$ in Eq. (3.4) to ensure the resulting methods to be total variation diminishing (TVD). These methods include the monotonic upstream-centered scheme for conservation laws (MUSCL) of van Leer and Minmod methods among others [28,51,98,99]. The simplest choice of the slope is probably the minmod slope defined by

$$
\sigma_{i}^{m}=\operatorname{minmod}\left\{\frac{\bar{c}_{i+1}^{m}-\bar{c}_{i}^{m}}{\Delta x}, \frac{\bar{c}_{i}^{m}-\bar{c}_{i-1}^{m}}{\Delta x}\right\}
$$

with $\operatorname{minmod}(a, b)=\frac{1}{2}(\operatorname{sgn}(a)+\operatorname{sgn}(b)) \min (|a|,|b|)$. 
In concluding this part, we notice the connection between the flux- and slope-limiter methods. Using formulation (3.3), we see that the numerical flux for the slope-limiter method (3.5) is

$$
F\left(c^{m}, i\right)=V c_{i}^{m}+\frac{\Delta x}{2} V(1-\mathrm{Cr}) \sigma_{i}^{m},
$$

which is of the same form as the flux-limiter method (3.3) if the slope-limiter $\sigma_{i}^{m}$ is related to the flux-limiter $\Psi\left(c^{m}, i\right)$ by $\sigma_{i}^{m}=\left[\left(c_{i+1}^{m}-c_{i}^{m}\right) / \Delta x\right] \Psi\left(c^{m}, i\right)$.

\subsubsection{Essentially nonoscillatory (ENO) schemes and weighted essentially nonoscillatory (WENO) schemes}

Traditional finite difference methods are based on fixed stencil interpolations of discrete data using polynomials. The resulting scheme is linear for linear PDEs. However, fixed stencil interpolation of second- or higher-order accuracy is necessarily oscillatory across a discontinuity; this is why the Lax-Wendroff scheme (2.8) and the Beam-Warming scheme (2.9) introduce oscillations across shock discontinuities (see Remark 3). One common approach to eliminate or reduce spurious oscillations near discontinuities is to add a numerical diffusion as in the SDFEM presented earlier. The numerical diffusion should be tuned so that it is large enough near discontinuities but is small enough elsewhere to maintain high-order accuracy. One disadvantage of this approach is that it is hard to determine an appropriate amount of numerical diffusion that introduces just enough dissipation without causing excessive smearing. Another approach is to apply (flux or slope) limiters to eliminate the oscillations. By carefully designing such limiters (e.g., reducing the slope of a linear interpolant or using a linear rather than a quadratic interpolant near shock discontinuities), the TVD property could be achieved for some numerical schemes for nonlinear scalar conservation laws in one space dimension. Unfortunately, Osher and Chakravarthy proved that TVD methods must degenerate to first-order accuracy at local maximum or minimum points [78].

The ENO and WENO schemes are high-order accurate finite difference/volume schemes designed for nonlinear hyperbolic conservation laws with piecewise smooth solutions containing discontinuities $[54,56,64,71]$. By delicately defining a nonlinear adaptive procedure to automatically choose the locally smooth stencil, the ENO and WENO schemes avoid crossing discontinuities in the interpolation procedure and thus generate uniformly high-order accurate, yet essentially nonoscillatory solutions. These schemes have been quite successful in applications, especially for problems containing both shock discontinuities and complicated smooth solution structures [92].

\subsubsection{The discontinuous Galerkin (DG) method}

The original discontinuous Galerkin finite element method was introduced by Reed and Hill for solving a linear neutron transport equation [84], in which the method can be carried out element by element when the elements are suitably ordered according to the characteristic directions. Lesaint and Raviart [69] carried out the first analysis for this method and proved a convergence rate of $(\Delta x)^{k}$ for general triangular partitions and $(\Delta x)^{k+1}$ for Cartesian grids. Johnson and Pitkaränta [65] obtained an improved estimate of $(\Delta x)^{k+1 / 2}$ for general triangulations, which is confirmed to be optimal by Peterson [79]. Chavent and Salzano [20] constructed an explicit DG method for Eq. (2.11), in which piecewise linear FEM is used in space and an explicit Euler approximation is used in time. Unfortunately, the scheme is stable only if the Courant number $\mathrm{Cr}=\mathrm{O}(\sqrt{\Delta x})$. Chavent and Cockburn [18] modified the scheme by introducing a slope limiter, and proved the scheme to be total variation 
bounded (TVB) when $\mathrm{Cr} \leqslant \frac{1}{2}$. However, the slope limiter introduced compromises the accuracy of the approximation in smooth regions. Cockburn and Shu [26] introduced the first Runge-Kutta DG (RKDG) method, which uses an explicit TVD second-order Runge-Kutta discretization and modifies the slope limiter to maintain the formal accuracy of the scheme at the extrema. The same authors then extended this approach to construct higher-order RKDG methods [25], to multidimensional scalar conservation laws [24,27], and to multidimensional systems. We refer interested readers to the survey article [23] in this volume for detailed discussions on the DG methods.

\subsection{Characteristic methods}

Because of the hyperbolic nature of advective transport, characteristic methods have been investigated extensively for the solution of advection-diffusion PDEs. In a characteristic (or Lagrangian) method, the transport of the fluid is referred to a Lagrangian coordinate system that moves with the fluid velocity. One tracks the movement of a fluid particle and the coordinate system follows the movement of the fluid. The time derivative along the characteristics of the advection-diffusion PDE (3.1) is expressed as

$$
\frac{D c}{D t}=\frac{\partial c}{\partial t}+\frac{\boldsymbol{u}}{\phi} \cdot \nabla c .
$$

Consequently, the advection-diffusion PDE (3.1) is rewritten as the following parabolic diffusionreaction PDE in a Lagrangian system:

$$
\phi \frac{D c}{D t}-\nabla \cdot(\boldsymbol{D} \nabla c)=\bar{c} q
$$

and the advection has seemingly disappeared. In other words, in a Lagrangian coordinate system (that moves with the flow) one would only see the effect of the diffusion, reaction, and the the right-hand side source terms but not the effect of the advection or moving steep fronts. Hence, the solutions of the advection-diffusion PDEs are much smoother along the characteristics than they are in the time direction. This explains why characteristic methods usually allow large time steps to be used in a numerical simulation while still maintaining its stability and accuracy. Unfortunately, Eq. (3.7) is written in a Lagrangian coordinate system, which is constantly moving in time. Consequently, the corresponding characteristic or Lagrangian methods often raise extra and nontrivial analytical, numerical, and implementational difficulties, which require very careful treatment. In contrast, Eq. (1.2) or (3.1) is written in an Eulerian system which is fixed in space. Hence, Eulerian methods are relatively easy to formulate and to implement.

\subsubsection{Classical characteristic or Eulerian-Lagrangian methods}

The classical Eulerian-Lagrangian method is a finite difference method based on the forward tracking of particles in cells. In this method, the spatial domain is divided into a collection of elements or cells and a number of particles are placed within each cell. Then the governing PDE is used to determine the movement of the particles from cell to cell. In this algorithm, the solution is determined by the number of particles within a cell at any given time. Related works can be found in $[47,49,96]$. In these methods, the diffusion occurs at the time step $t^{m}$ and the solution is advected 
forward in time to the time step $t^{m+1}$, leading to the following scheme for Eq. (2.1):

$$
\frac{\tilde{c}_{i}^{m+1}-c_{i}^{m}}{\Delta t}-D \frac{c_{i+1}^{m}-2 c_{i}^{m}+c_{i-1}^{m}}{(\Delta x)^{2}}=0 .
$$

Here $\tilde{c}_{i}^{m+1}=c\left(\tilde{x}_{i}, t^{m+1}\right)$ with $\tilde{x}_{i}=x_{i}+V \Delta t$. Because the advected nodes $\tilde{x}_{i}$ need not be nodes at time $t^{m+1}$, they are irregular, in general.

Neuman developed an Eulerian-Lagrangian finite element method using a combination of forward and backward tracking algorithms [76,77]. Near a steep front, a forward tracking algorithm is used to move a cloud of particles from time $t^{m}$ to new positions at time $t^{m+1}$ according to the advection, as done by Garder et al. [49]. An implicit scheme is then used to treat the diffusion at time $t^{m+1}$. Away from a front, a backward tracking algorithm is used, in which one finds a point that ends up at position $x$ at time $t^{m+1}$.

Eulerian methods carry out the temporal discretization in the time direction, so they cannot accurately simulate all of the wave interactions that take place if the information propagates more than one cell per time step (i.e., if the CFL condition is violated), either for the reason of stability (for explicit methods) or for the reason of accuracy (for implicit methods). By using characteristic tracking, characteristic methods follow the movement of information or particles as well as their interactions. However, forward tracked characteristic methods often distort the evolving grids severely and greatly complicate the solution procedures, especially for multi-dimensional problems.

\subsubsection{The modified method of characteristics (MMOC)}

In this part we briefly review the MMOC, which was proposed by Douglas and Russell for solving advection-diffusion PDEs in a nonconservative form [37] and can be viewed as a representative of the Eulerian-Lagrangian methods developed during the same time period $[6,80,82]$. Using the Lagrangian form (3.7), we can combine the first two terms on the left-hand side of (3.1) to form one term through characteristic tracking (3.6) (see, e.g. [37])

$$
\phi \frac{D c\left(\boldsymbol{x}, t^{m+1}\right)}{D t} \approx \phi(\boldsymbol{x}) \frac{c\left(\boldsymbol{x}, t^{m+1}\right)-c\left(\boldsymbol{x}^{*}, t^{m}\right)}{\Delta t}
$$

with $\boldsymbol{x}^{*}=\boldsymbol{x}-\boldsymbol{u}\left(\boldsymbol{x}, t^{m+1}\right) \Delta t / \phi(\boldsymbol{x})$.

Substituting (3.8) for the first two terms on the left-hand side of Eq. (3.1) and integrating the resulting equation against any finite element test functions $w(\boldsymbol{x})$, one obtains the following MMOC scheme [37,43] for Eq. (3.1):

$$
\begin{aligned}
\int_{\Omega} \phi(\boldsymbol{x}) \frac{c\left(\boldsymbol{x}, t^{m+1}\right)-c\left(\boldsymbol{x}^{*}, t^{m}\right)}{\Delta t} w(\boldsymbol{x}) \mathrm{d} \boldsymbol{x}+\int_{\Omega} \nabla w(\boldsymbol{x}) \cdot \boldsymbol{D} \nabla c\left(\boldsymbol{x}, t^{m+1}\right) \mathrm{d} \boldsymbol{x} \\
\quad=\int_{\Omega} \bar{c} q\left(\boldsymbol{x}, t^{m+1}\right) w(\boldsymbol{x}) \mathrm{d} \boldsymbol{x} .
\end{aligned}
$$

Eq. (3.9) follows the flow by tracking the characteristics backward from a point $\boldsymbol{x}$ in a fixed grid at the time step $t^{m+1}$ to a point $\boldsymbol{x}^{*}$ at time $t^{m}$. Hence, the MMOC avoids the grid distortion problems present in forward tracking methods. Moreover, MMOC symmetrizes and stabilizes the transport 
PDEs, greatly reducing temporal errors; therefore MMOC allows for large time steps in a simulation without loss of accuracy and eliminates the excessive numerical dispersion and grid orientation effects present in many Eulerian methods [36,40,89]. However, the MMOC and the characteristic methods presented earlier have the following drawbacks:

Remark 4. In the context of the MMOC and other characteristic methods using a backtracking algorithm, the $\int_{\Omega} \phi(\boldsymbol{x}) c\left(\boldsymbol{x}^{*}, t^{m}\right) w(\boldsymbol{x}) \mathrm{d} \boldsymbol{x}$ term in Eq. (3.9) is defined on the domain at time $t^{m+1}$. In this term, the test functions $w(\boldsymbol{x})$ are standard FEM basis functions on $\Omega$ at time $t^{m+1}$, but the value of $c\left(\boldsymbol{x}^{*}, t^{m}\right)$ has to be evaluated by a backtracking method where $\boldsymbol{x}^{*}=\boldsymbol{r}\left(t^{m} ; \boldsymbol{x}, t^{m+1}\right)$ is the point at the foot corresponding to $\boldsymbol{x}$ at the head [37,43]. For multidimensional problems, the evaluation of this term with a backtracking algorithm requires significant effort, due to the need to define the geometry at time $t^{m}$ that requires mapping of points along the boundary of the element and subsequent interpolation and mapping onto the fixed spatial grid at the previous time $t^{m}[7,74]$. This procedure introduces a mass balance error and leads to schemes that fail to conserve mass $[15,74,107]$. Moreover, in these methods it is not clear how to treat flux boundary conditions in a mass-conservative manner without compromising the accuracy, when the characteristics track to the boundary of the domain $[15,88,104,106,107]$.

\subsubsection{The modified method of characteristics with adjusted advection (MMOCAA)}

Recently, Douglas et al. proposed an MMOCAA scheme to correct the mass error of the MMOC by perturbing the foot of the characteristics slightly [34,35]. For Eq. (3.1) with a no-flow or periodic boundary condition, the summation of Eq. (3.9) for all the test functions (that add exactly to one) yields the following equation:

$$
\int_{\Omega} \phi(\boldsymbol{x}) c\left(\boldsymbol{x}, t^{m+1}\right) \mathrm{d} \boldsymbol{x}-\int_{\Omega} \phi(\boldsymbol{x}) c\left(\boldsymbol{x}^{*}, t^{m}\right) \mathrm{d} \boldsymbol{x}=\Delta t \int_{\Omega} \bar{c} q\left(\boldsymbol{x}, t^{m+1}\right) \mathrm{d} \boldsymbol{x} .
$$

Recall that the term on the right-hand side of this equation is obtained by an Euler approximation to the temporal integral in this term. On the other hand, integrating the original PDE (1.2) on the domain $\Omega \times\left[t^{m}, t^{m+1}\right]$ yields the following equation:

$$
\int_{\Omega} \phi(\boldsymbol{x}) c\left(\boldsymbol{x}, t^{m+1}\right) \mathrm{d} \boldsymbol{x}-\int_{\Omega} \phi(\boldsymbol{x}) c\left(\boldsymbol{x}, t^{m}\right) \mathrm{d} \boldsymbol{x}=\int_{t^{m}}^{t^{m+1}} \int_{\Omega} \bar{c} q \mathrm{~d} \boldsymbol{x} \mathrm{d} t .
$$

Therefore, to maintain mass balance, we must have

$$
\int_{\Omega} \phi(\boldsymbol{x}) c\left(\boldsymbol{x}, t^{m}\right) \mathrm{d} \boldsymbol{x} \equiv Q^{m}=Q_{*}^{m} \equiv \int_{\Omega} \phi(\boldsymbol{x}) c\left(\boldsymbol{x}^{*}, t^{m}\right) \mathrm{d} \boldsymbol{x} .
$$

For some fixed constant $\kappa>0$, we define

$$
\begin{aligned}
& \boldsymbol{x}_{+}^{*}=\boldsymbol{x}-\frac{\boldsymbol{u}\left(\boldsymbol{x}, t^{m+1}\right)}{\phi(\boldsymbol{x})} \Delta t+\kappa \frac{\boldsymbol{u}\left(\boldsymbol{x}, t^{m+1}\right)}{\phi(\boldsymbol{x})}(\Delta t)^{2}, \\
& \boldsymbol{x}_{-}^{*}=\boldsymbol{x}-\frac{\boldsymbol{u}\left(\boldsymbol{x}, t^{m+1}\right)}{\phi(\boldsymbol{x})} \Delta t-\kappa \frac{\boldsymbol{u}\left(\boldsymbol{x}, t^{m+1}\right)}{\phi(\boldsymbol{x})}(\Delta t)^{2} .
\end{aligned}
$$


We also define

$$
c_{\#}\left(\boldsymbol{x}^{*}, t^{m}\right)= \begin{cases}\max \left\{c\left(\boldsymbol{x}_{+}^{*}, t^{m}\right), c\left(\boldsymbol{x}_{-}^{*}, t^{m}\right)\right\}, & \text { if } Q_{*}^{m} \leqslant Q^{m}, \\ \min \left\{c\left(\boldsymbol{x}_{+}^{*}, t^{m}\right), c\left(\boldsymbol{x}_{-}^{*}, t^{m}\right)\right\}, & \text { if } Q_{*}^{m}>Q^{m} .\end{cases}
$$

Because $c\left(\boldsymbol{x}, t^{m+1}\right)$ is unknown in the evaluation of $Q^{m}$, an extrapolation of $2 c\left(\boldsymbol{x}, t^{m}\right)-c\left(\boldsymbol{x}, t^{m-1}\right)$ is used. We set

$$
Q_{\#}^{m}=\int_{\Omega} \phi(\boldsymbol{x}) c_{\#}\left(\boldsymbol{x}^{*}, t^{m}\right) \mathrm{d} \boldsymbol{x} .
$$

If $Q_{\#}^{m}=Q_{*}^{m}$, we let $\breve{c}\left(\boldsymbol{x}^{*}, t^{m}\right)=c\left(\boldsymbol{x}^{*}, t^{m}\right)$. In this case, the mass is not conserved. Otherwise, find $\theta^{m}$ such that $Q^{m}=\theta^{m} Q_{*}^{m}+\left(1-\theta^{m}\right) Q_{\#}^{m}$ and let $\breve{c}\left(\boldsymbol{x}^{*}, t^{m}\right)=\theta^{m} c\left(\boldsymbol{x}^{*}, t^{m}\right)+(1-\theta) c_{\#}\left(\boldsymbol{x}^{*}, t^{m}\right)$. In latter case, one has

$$
\int_{\Omega} \phi(\boldsymbol{x}) \check{c}\left(\boldsymbol{x}^{*}, t^{m}\right) \mathrm{d} \boldsymbol{x}=Q^{m} .
$$

Hence, mass is conserved globally. In the MMOCAA procedure one replaces $c\left(\boldsymbol{x}^{*}, t^{m}\right)$ in (3.8) and (3.9) by $\check{c}\left(\boldsymbol{x}^{*}, t^{m}\right)$.

\subsubsection{The Eulerian-Lagrangian localized adjoint method (ELLAM)}

The ELLAM formalism was introduced by Celia et al. for the solution of one-dimensional advection-diffusion PDEs [16,60]. It provides a general characteristic solution procedure for advectiondominated PDEs, and it presents a consistent framework for treating general boundary conditions and maintaining mass conservation. The ELLAM formulation directly applies to Eq. (1.2) in a conservative form. Multiplying Eq. (1.2) with space-time test functions $w$ that vanish outside $\Omega \times\left(t^{m}, t^{m+1}\right]$ and are discontinuous in time at time $t^{m}$, and integrating the resulting equation over the space-time domain $\Omega \times\left(t^{m}, t^{m+1}\right.$, we obtain a space-time weak formulation for Eq. (1.2) with a noflow boundary condition

$$
\begin{gathered}
\int_{\Omega} \phi(\boldsymbol{x}) c\left(\boldsymbol{x}, t^{m+1}\right) w\left(\boldsymbol{x}, t^{m+1}\right) \mathrm{d} \boldsymbol{x}+\int_{t^{m}}^{t^{m+1}} \int_{\Omega} \nabla w \cdot(\boldsymbol{D} \nabla c) \mathrm{d} \boldsymbol{x} \mathrm{d} t \\
\quad-\int_{t^{m}}^{t^{m+1}} \int_{\Omega} c \rho\left(\phi w_{t}+\boldsymbol{u} \cdot \nabla w\right) \mathrm{d} \boldsymbol{x} \mathrm{d} t \\
=\int_{\Omega} \phi(\boldsymbol{x}) c\left(\boldsymbol{x}, t^{m}\right) w\left(\boldsymbol{x}, t_{+}^{m}\right) \mathrm{d} \boldsymbol{x}+\int_{t^{m}}^{t^{m+1}} \int_{\Omega} \bar{c} q w \mathrm{~d} \boldsymbol{x} \mathrm{d} t,
\end{gathered}
$$

where $w\left(\boldsymbol{x}, t_{+}^{m}\right)=\lim _{t \rightarrow t_{+}^{m}} w(\boldsymbol{x}, t)$ takes into account that $w(x, t)$ is discontinuous in time at time $t^{m}$.

Motivated by the localized adjoint method, the ELLAM formalism chooses the test functions from the solution space of the homogeneous adjoint equation of Eq. (1.2) (e.g. see $[16,60]$ )

$$
-\phi(\boldsymbol{x}) \frac{\partial w}{\partial t}-\boldsymbol{u} \cdot \nabla w-\nabla \cdot(\boldsymbol{D} \nabla w)=0
$$

Because the solution space for Eq. (3.11) is infinite dimensional and only a finite number of test functions should be used, an operator splitting technique is applied to Eq. (3.11) to define the test functions. 
(i) In the first splitting, the two terms involving spatial derivatives are grouped together, leading to the following system of equations:

$$
\begin{aligned}
& -\phi(\boldsymbol{x}) \frac{\partial w}{\partial t}=0, \\
& -\boldsymbol{u} \cdot \nabla w-\nabla \cdot(\boldsymbol{D} \nabla w)=0 .
\end{aligned}
$$

This splitting leads to a class of optimal test function methods involving upstream weighting in space $[4,15,22]$, which yield solutions with significant temporal errors and numerical diffusion.

(ii) In the second splitting, the terms involving first-order derivatives are grouped together, leading to the following system of equations:

$$
\begin{aligned}
& -\phi(\boldsymbol{x}) \frac{\partial w}{\partial t}-\boldsymbol{u} \cdot \nabla w=0, \\
& -\nabla \cdot(\boldsymbol{D} \nabla w)=0 .
\end{aligned}
$$

The first equation in (3.12) implies that the test functions should be constant along the characteristics defined by

$$
\frac{\mathrm{d} \boldsymbol{r}}{\mathrm{d} t}=\frac{\boldsymbol{u}(\boldsymbol{r}, \theta)}{\phi(\boldsymbol{r})}
$$

which reflects the hyperbolic nature of Eq. (1.2) and assures Lagrangian treatment of advection. The second equation in (3.13) is an elliptic PDE, so standard FEM approximations would be a natural choice for the spatial configuration of the test functions.

Using splitting (3.12), we define the test functions to be standard FEM basis functions on the spatial domain $\bar{\Omega}$ at time $t^{m+1}$ and extend them by a constant into the space-time strip $\bar{\Omega} \times\left[t^{m}, t^{m+1}\right]$ along the characteristics defined by (3.13). Incorporating these test functions into the reference equation (3.10), we obtain an ELLAM scheme as follows:

$$
\begin{aligned}
\int_{\Omega} \phi(\boldsymbol{x}) c\left(\boldsymbol{x}, t^{m+1}\right) \mathrm{d} \boldsymbol{x}+\Delta t \int_{\Omega}(\nabla w \cdot(\boldsymbol{D} \nabla c))\left(\boldsymbol{x}, t^{m+1}\right) \mathrm{d} \boldsymbol{x} \\
=\int_{\Omega} \phi(\boldsymbol{x}) c\left(\boldsymbol{x}, t^{m}\right) w\left(\boldsymbol{x}, t_{+}^{m}\right) \mathrm{d} \boldsymbol{x}+\Delta t \int_{\Omega}(\bar{c} q w)\left(\boldsymbol{x}, t^{m+1}\right) \mathrm{d} \boldsymbol{x} .
\end{aligned}
$$

Remark 5. The ELLAM scheme (3.14) symmetrizes the transport PDE (1.2), and generates accurate numerical solutions without excessive numerical diffusion or nonphysical oscillation even if coarse spatial grids and large time steps are used [87,104,106]. Second, it is proved that the ELLAM scheme conserves mass [16,88]. Third, in contrast to the MMOC and many other characteristic methods that treat general boundary conditions in an ad hoc manner, the ELLAM formulation can treat any combinations of boundary conditions and provides a systematic way to calculate the boundary conditions accurately $[13,16,104,106]$. Thus, the ELLAM formulation overcomes the drawbacks of many previous characteristic methods while maintaining their numerical advantages.

Remark 6. Most integrals in the ELLAM scheme (3.14) are standard in FEMs and can be evaluated in a straightforward manner. The only exception is the $\int_{\Omega} \phi(\boldsymbol{x}) c\left(\boldsymbol{x}, t^{m}\right) w\left(\boldsymbol{x}, t_{+}^{m}\right) \mathrm{d} \boldsymbol{x}$ term on the right-hand side of Eq. (3.14). This term corresponds to the $\int_{\Omega} \phi(\boldsymbol{x}) c\left(\boldsymbol{x}^{*}, t^{m}\right) w(\boldsymbol{x}) \mathrm{d} \boldsymbol{x}$ term in the 
MMOC scheme (3.9). As discussed in Remark 4, in the MMOC and other characteristic methods using a backtracking algorithm the evaluation of the $\int_{\Omega} \phi(\boldsymbol{x}) c\left(\boldsymbol{x}^{*}, t^{m}\right) w(\boldsymbol{x}) \mathrm{d} \boldsymbol{x}$ term requires significant effort and introduces mass balance error [7,74]. In the ELLAM scheme (3.14), the term $\int_{\Omega} \phi(\boldsymbol{x}) c\left(\boldsymbol{x}, t^{m}\right) w\left(\boldsymbol{x}, t_{+}^{m}\right) \mathrm{d} \boldsymbol{x}$ is evaluated by a forward tracking algorithm that was proposed by Russell and Trujillo [88]. In this approach, an integration quadrature would be enforced on $\bar{\Omega}$ at time $t^{m}$ with respect to a fixed spatial grid on which $c\left(\boldsymbol{x}, t^{m}\right)$ is defined. The difficult evaluation of $w\left(\boldsymbol{x}, t_{+}^{m}\right)=\lim _{t \rightarrow t_{+}^{m}} w\left(\boldsymbol{x}, t^{m}\right)=w\left(\tilde{\boldsymbol{x}}, t^{m+1}\right)$ is carried out by a forward tracking algorithm from $\boldsymbol{x}$ at time $t^{m}$ to $\tilde{\boldsymbol{x}}=\boldsymbol{r}\left(t^{m+1} ; \boldsymbol{x}, t^{m}\right)$ at time $t^{m+1}$. Because this forward tracking is used only in the evaluation of the right-hand side of (3.14), it has no effect on the solution grid or the data structure of the discrete system. Therefore, the forward tracking algorithm used here does not suffer from the complication of distorted grids, which complicates many classical forward tracking algorithms.

In the past few years, Wang et al. developed ELLAM schemes for multidimensional advectiondiffusion PDEs [101,104,109]; Ewing and Wang [45] and Wang et al. [106] also developed ELLAM schemes for multidimensional advection-reaction PDEs; Celia and Ferrand [14] and Healy and Russell [57,58] developed ELLAM schemes in a finite-volume setting. Dahle et al. developed ELLAM for two-phase flow [33,42]. The computational experiments carried out in $[104,106]$ showed that the ELLAM schemes often outperform many widely used and well-received numerical methods in the context of linear advection-diffusion or advection-reaction PDEs. In addition, Binning and Celia developed a backtracking finite-volume ELLAM scheme for unsaturated flow [7], Wang et al. developed an ELLAM-MFEM solution technique for porous medium flows with point sources and sinks [108]. These works illustrate the strength of the ELLAM schemes in solving the coupled systems of advection-diffusion PDEs. From a viewpoint of analysis, ELLAM methods introduce further difficulties and complexities to the already complicated analyses of characteristic methods. We refer readers to the works of Wang et al. for the convergence analysis and optimal-order error estimates for the ELLAM schemes for advection-diffusion or advection-diffusion-reaction PDEs [102,103,105,107], and the corresponding analysis of Ewing and Wang for the ELLAM schemes for advection-reaction PDEs [44-46].

\subsubsection{The characteristic mixed finite element method (CMFEM)}

The CMFEM was presented by Arbogast et al. in [2,3] and Yang in [113], and can be viewed as a procedure of ELLAM type [3]. It is also based on the space-time weak formulation (3.10), but uses a mixed finite element approach by introducing the diffusive flux $\boldsymbol{z}=-\boldsymbol{D} \nabla$ as a new variable. Let $\boldsymbol{V}_{h} \times W_{h}$ be the lowest-order Raviart-Thomas spaces [83], and $\hat{W}_{h}$ be the space of discontinuous piecewise-linear functions on the same partition. Then, the CMFEM scheme can be formulated as follows: find $c\left(\boldsymbol{x}, t^{m+1}\right) \in W_{h}$ and $\boldsymbol{z}\left(\boldsymbol{x}, t^{m+1}\right) \in \mathscr{V}_{h}$ such that

$$
\begin{gathered}
\int_{\Omega} \phi(\boldsymbol{x}) \frac{c\left(\boldsymbol{x}, t^{m+1}\right)-\hat{c}\left(\boldsymbol{x}^{*}, t^{m}\right)}{\Delta t} w(\boldsymbol{x}) \mathrm{d} \boldsymbol{x}+\int_{\Omega} \nabla \cdot \boldsymbol{z}\left(\boldsymbol{x}, t^{m+1}\right) w(\boldsymbol{x}) \mathrm{d} \boldsymbol{x} \\
=\int_{\Omega}(\bar{c}-c) q\left(\boldsymbol{x}, t^{m+1}\right) w(\boldsymbol{x}) \mathrm{d} \boldsymbol{x}, \quad \forall w \in W_{h}, \\
\int_{\Omega} \boldsymbol{D}^{-1} \boldsymbol{z}\left(\boldsymbol{x}, t^{m+1}\right) \mathrm{d} \boldsymbol{x}-\int_{\Omega} c\left(\boldsymbol{x}, t^{m+1}\right) \nabla \cdot \boldsymbol{v} \mathrm{d} \boldsymbol{x}=0, \quad \forall \boldsymbol{v} \in \boldsymbol{V}_{h},
\end{gathered}
$$


where $\boldsymbol{x}^{*}$ is defined in (3.8) and $\hat{c}\left(\boldsymbol{x}, t^{m}\right) \in \hat{W}_{h}$ is a post-processing of $c\left(\boldsymbol{x}, t^{m}\right)$ and $\boldsymbol{z}\left(\boldsymbol{x}, t^{m}\right)$ defined by

$$
\begin{aligned}
& \int_{\Omega} \phi(\boldsymbol{x}) \hat{c}\left(\boldsymbol{x}, t^{m}\right) w(x) \mathrm{d} \boldsymbol{x}=\int_{\Omega} c\left(\boldsymbol{x}, t^{m}\right) w(\boldsymbol{x}) \mathrm{d} \boldsymbol{x}, \quad \forall w \in W_{h}, \\
& \int_{\Omega} \nabla \hat{w}(\boldsymbol{x}) \cdot(\boldsymbol{D} \nabla \hat{c})\left(\boldsymbol{x}, t^{m}\right) \mathrm{d} \boldsymbol{x}=-\int_{\Omega} \boldsymbol{z}\left(\boldsymbol{x}, t^{m}\right) \hat{w}(\boldsymbol{x}) \mathrm{d} \boldsymbol{x}, \quad \forall \hat{w} \in \hat{W}_{h} .
\end{aligned}
$$

It is well known that in the mixed method, the scalar variable $c\left(\boldsymbol{x}, t^{m+1}\right)$ is of first order accuracy in space. This post-processing procedure is used to improve the accuracy to the order of $\mathrm{O}\left((\Delta x)^{3 / 2}\right)[3]$

Remark 7. Theoretically the CMFEM is locally mass conservative. The situation might not be so clear numerically due to the following reasons: (i) The post-processing procedure is anti-diffusive and, hence, could yield $\hat{c}$ with undershoot or overshoot. A slope limiter has been used in the implementation of CMFEM to overcome this problem [2]. It is not clear how the local mass conservation is achieved in this case. (ii) The CMFEM inherently requires a backtracking procedure and thus has to exactly determine the backtracked image at the previous time step $t^{m}$ of each cell at the future time step $t^{m+1}$ in order to conserve mass. Since the backtracked image of each cell typically has curved boundaries in general, it is not clear how to trace these cell boundaries exactly to conserve mass numerically. Finally, the theoretically proved error estimate for the CMFEM is obtained only for Eq. (1.2) with a periodic boundary condition and is of $\mathrm{O}\left((\Delta x)^{3 / 2}\right)$ which is suboptimal by a factor $\mathrm{O}\left((\Delta x)^{1 / 2}\right)$.

\subsubsection{Characteristic methods for immiscible fluid flows, operator splitting techniques}

In the governing equation (1.5) for immiscible flows, the hyperbolic part is given by Eq. (2.11) with a typically $S$-shaped function of the unknown, while the unknown function is a decreasing function in space. Hence, Eq. (2.11) could develop a non-unique solution [12,48,67,70]. Thus, characteristic methods do not apply directly. Espedal and Ewing [38] presented an operator-splitting technique to overcome this difficulty. The fractional flow function $f(c)$ is split into an advective concave hull $\bar{f}(c)$ of $f(c)$, which is linear in what would be the shock region of Eq. (2.11), and a residual anti-diffusive part. The modified advection PDE

$$
\frac{\partial c}{\partial t}+\frac{\partial \bar{f}(c)}{\partial x}=0, \quad \boldsymbol{x} \in \Omega, t \in[0, T]
$$

yields the same entropy solution as the PDE (2.11), and thus defines characteristic directions uniquely. The residual anti-diffusive advection term is grouped with the diffusion term in the governing PDE so that correct balance between nonlinear advection and diffusion is obtained. Numerically, the PDE is solved by a quadratic Petrov-Galerkin FEM. This technique has been applied in numerical simulation for immiscible flow by Espedal, Ewing, and their collaborators [32,39]. Subsequently, Ewing [41] and Dahle et al. have applied the operator-splitting technique to develop an ELLAM scheme for nonlinear advection-diffusion PDEs, which has shown very promising results. 


\section{References}

[1] D. Allen, R. Southwell, Relaxation methods applied to determining the motion in two dimensions of a fluid past a fixed cylinder, Quart. J. Mech. Appl. Math. 8 (1955) 129-145.

[2] T. Arbogast, A. Chilakapati, M.F. Wheeler, A characteristic-mixed method for contaminant transport and miscible displacement, in: T.F. Russell, R.E. Ewing, C.A. Brebbia, W.G. Gray and G.F. Pinder (Eds.), Computational Methods in Water Resources IX, Vol. 1, Computational Mechanics Publications and Elsevier Applied Science, London and New York, 1992, pp. 77-84.

[3] T. Arbogast, M.F. Wheeler, A characteristic-mixed finite element method for advection-dominated transport problems, SIAM J. Numer. Anal. 32 (1995) 404-424.

[4] J.W. Barrett, K.W. Morton, Approximate symmetrization and Petrov-Galerkin methods for diffusion-convection problems, Comput. Methods Appl. Mech. Eng. 45 (1984) 97-122.

[5] J. Bear, Hydraulics of Groundwater, McGraw-Hill, New York, 1979.

[6] J.P. Benque, J. Ronat, Quelques difficulties des modeles numeriques en hydraulique, in: R. Glowinski, J.-L. Lions (Eds.), Computer Methods in Applied Mechanics and Engineering, North-Holland, Amsterdam, 1982, pp. 471-494.

[7] P.J. Binning, M.A. Celia, A finite volume Eulerian-Lagrangian localized adjoint method for solution of the contaminant transport equations in two-dimensional multi-phase flow systems, Water Resour. Res. 32 (1996) 103-114.

[8] J.P. Boris, D.L. Book, Flux-corrected transport I, SHASTA, a fluid transport algorithm that works, J. Comput. Phys. 11 (1973) 38-69.

[9] J.P. Boris, D.L. Book, Flux-corrected transport, III, Minimal-error FCT algorithms, J. Comput. Phys. 20 (1976) 397-431.

[10] E.T. Bouloutas, M.A. Celia, An improved cubic Petrov-Galerkin method for simulation of transient advectiondiffusion processes in rectangularly decomposable domains, Comput. Meth. Appl. Mech. Eng. 91 (1991) $289-308$.

[11] A. Brooks, T.J.R. Hughes, Streamline upwind Petrov-Galerkin formulations for convection dominated flows with particular emphasis on the incompressible Navier-Stokes equations, Comput. Meth. Appl. Mech. Eng. 32 (1982) 199-259.

[12] S.E. Buckley, M.C. Leverret, Mechanism of fluid displacement in sands, Trans. AIME 146 (1942) $107-116$.

[13] M.A. Celia, Eulerian-Lagrangian localized adjoint methods for contaminant transport simulations, in: Peters et al. (Eds.), Computational Methods in Water Resources X, Vol. 1, Water Science and Technology Library, Vol. 12, Kluwer Academic Publishers, Dordrecht, Netherlands, 1994, pp. 207-216.

[14] M.A. Celia, L.A. Ferrand, A comparison of ELLAM formulations for simulation of reactive transport in groundwater, in: Wang (Ed.), Advances in Hydro-Science and Engineering, Vol. 1(B), University of Mississippi Press, Mississippi, 1993, pp. 1829-1836.

[15] M.A. Celia, I. Herrera, E.T. Bouloutas, J.S. Kindred, A new numerical approach for the advective-diffusive transport equation, Numer. Meth. PDEs 5 (1989) 203-226.

[16] M.A. Celia, T.F. Russell, I. Herrera, R.E. Ewing, An Eulerian-Lagrangian localized adjoint method for the advection-diffusion equation, Adv. Water Resour. 13 (1990) 187-206.

[17] G. Chavent, A new formulation of diphasic incompressible flows in porous media, in: Lecture Notes in Mathematics, Vol. 503, Springer, Berlin, 1976.

[18] G. Chavent, B. Cockburn, The local projection $P^{0}, P^{1}$-discontinuous Galerkin finite element method for scalar conservation laws, $\mathrm{M}^{2} \mathrm{AN} 23$ (1989) 565-592.

[19] G. Chavent, J. Jaffré, Mathematical Models and Finite Elements for Reservoir Simulation, North-Holland, Amsterdam, 1986.

[20] G. Chavent, G. Salzano, A finite element method for the 1D water flooding problem with gravity, J. Comput. Phys. 45 (1982) 307-344.

[21] Z. Chen, R.E. Ewing, Comparison of various formulations of three-phase flow in porous media, J. Comput. Phys. 132 (1997) 362-373.

[22] I. Christie, D.F. Griffiths, A.R. Mitchell, O.C. Zienkiewicz, Finite element methods for second order differential equations with significant first derivatives, Int. J. Numer. Eng. 10 (1976) 1389-1396.

[23] B. Cockburn, Finite element methods for conservation laws, this volume, J. Comput. Appl. Math. 128 (2001) 187-204. 
[24] B. Cockburn, S. Hou, C.-W. Shu, TVB Runge-Kutta local projection discontinuous Galerkin finite element method for conservation laws IV: the multidimensional case, Math. Comp. 54 (1990) 545-590.

[25] B. Cockburn, C.-W. Shu, The Runge-Kutta local projection $P^{1}$-discontinuous Galerkin finite element method for scalar conservation laws II: general framework, Math. Comp. 52 (1989) 411-435.

[26] B. Cockburn, C.-W. Shu, The Runge-Kutta local projection $P^{1}$-discontinuous Galerkin finite element method for scalar conservation laws, $\mathrm{M}^{2}$ AN 25 (1991) 337-361.

[27] B. Cockburn, C.-W. Shu, The Runge-Kutta discontinuous Galerkin finite element method for conservation laws V: multidimensional systems, J. Comput. Phys. 141 (1998) 199-224.

[28] P. Colella, A direct Eulerian MUSCL scheme for gas dynamics, SIAM J. Sci. Statist. Comput. 6 (1985) 104-117.

[29] P. Colella, P. Woodward, The piecewise-parabolic method (PPM) for gasdynamical simulations, J. Comput. Phys. 54 (1984) 174-201.

[30] R. Courant, K.O. Friedrichs, H. Lewy, Uber die partiellen differenzen-gleichungen der mathematisches physik, Math. Ann. 100 (1928) 32-74.

[31] R. Courant, E. Isaacson, M. Rees, On the solution of nonlinear hyperbolic differential equations by finite differences, Comm. Pure. Appl. Math. 5 (1952) 243-255.

[32] H.K. Dahle, M.S. Espedal, R.E. Ewing, O. Sævareid, Characteristic adaptive sub-domain methods for reservoir flow problems, Numer. Methods PDE's 6 (1990) 279-309.

[33] H.K. Dahle, R.E. Ewing, T.F. Russell, Eulerian-Lagrangian localized adjoint methods for a nonlinear convection-diffusion equation, Comput. Methods Appl. Mech. Eng. 122 (1995) 223-250.

[34] J. Douglas Jr., F. Furtado, F. Pereira, On the numerical simulation of waterflooding of heterogeneous petroleum reservoirs, Comput. Geosci. 1 (1997) 155-190.

[35] J. Douglas Jr., C.-S. Huang, F. Pereira, The modified method of characteristics with adjusted advection, Technical Report, No. 298, Center for Applied Mathematics, Purdue University, 1997.

[36] J. Douglas Jr., I. Martinéz-Gamba, M.C.J. Squeff, Simulation of the transient behavior of a one-dimensional semiconductor device, Mat. Apl. Comput. 5 (1986) 103-122.

[37] J. Douglas Jr., T.F. Russell, Numerical methods for convection-dominated diffusion problems based on combining the method of characteristics with finite element or finite difference procedures, SIAM J. Numer. Anal. 19 (1982) $871-885$.

[38] M.S. Espedal, R.E. Ewing, Characteristic Petrov-Galerkin sub-domain methods for two-phase immiscible flow, Comput. Methods Appl. Mech. Eng. 64 (1987) 113-135.

[39] M.S. Espedal, R.E. Ewing, J.A. Puckett, R.S. Schmidt, Simulation techniques for multiphase and multicomponent flows, Comm. Appl. Numer. Methods 4 (1988) 335-342.

[40] R.E. Ewing (Ed.), The Mathematics of Reservoir Simulation, Research Frontiers in Applied Mathematics, Vol. 1, SIAM, Philadelphia, PA, 1984.

[41] R.E. Ewing, Operator splitting and Eulerian-Lagrangian localized adjoint methods for multi-phase flow, in: Whiteman (Ed.), The Mathematics of Finite Elements and Applications VII, MAFELAP, 1990, Academic Press, San Diego, CA, 1991, pp. 215-232.

[42] R.E. Ewing, Simulation of multiphase flows in porous media, Transport in Porous Media 6 (1991) 479-499.

[43] R.E. Ewing, T.F. Russell, M.F. Wheeler, Simulation of miscible displacement using mixed methods and a modified method of characteristics, SPE 122411983 (71-81).

[44] R.E. Ewing, H. Wang, Eulerian-Lagrangian localized adjoint methods for linear advection equations, in: Computational Mechanics '91, Springer, Berlin, 1991, pp. 245-250.

[45] R.E. Ewing, H. Wang, Eulerian-Lagrangian localized adjoint methods for linear advection or advection-reaction equations and their convergence analysis, Comput. Mech. 12 (1993) 97-121.

[46] R.E. Ewing, H. Wang, An optimal-order error estimate to Eulerian-Lagrangian localized adjoint method for variable-coefficient advection-reaction problems, SIAM Numer. Anal. 33 (1996) 318-348.

[47] C.L. Farmer, A moving point method for arbitrary Peclet number multi-dimensional convection-diffusion equations, IMA J. Numer. Anal. 5 (1980) 465-480.

[48] B.A. Finlayson, Numerical Methods for Problems with Moving Fronts, Ravenna Park Publishing, Seattle, 1992.

[49] A.O. Garder, D.W. Peaceman, A.L. Pozzi, Numerical calculations of multidimensional miscible displacement by the method of characteristics, Soc. Pet. Eng. J. 4 (1964) 26-36. 
[50] S.K. Godunov, A difference scheme for numerical computation of discontinuous solutions of fluid dynamics, Mat. Sb. 47 (1959) 271-306.

[51] J.B. Goodman, R.J. LeVeque, A geometric approach to high-resolution TVD schemes, SIAM J. Numer. Anal. 25 (1988) 268-284.

[52] P. Hansbo, A. Szepessy, A velocity-pressure streamline diffusion finite element method for the incompressible Navier-Stokes equations, Comput. Methods Appl. Mech. Eng. 84 (1990) 107-129.

[53] A. Harten, High resolution schemes for hyperbolic conservation laws, J. Comput. Phys. 49 (1983) 357-393.

[54] A. Harten, B. Engquist, S. Osher, S. Chakravarthy, Uniformly high-order accurate essentially nonoscillatory schemes, III, J. Comput. Phys. 71 (1987) 231-241.

[55] A. Harten, J.M. Hyman, P.D. Lax, On finite-difference approximations and entropy conditions for shocks, Comm. Pure Appl. Math. 29 (1976) 297-322.

[56] A. Harten, S. Osher, Uniformly high-order accurate nonoscillatory schemes, I, SIAM J. Numer. Anal. 24 (1987) 279-309.

[57] R.W. Healy, T.F. Russell, A finite-volume Eulerian-Lagrangian localized adjoint method for solution of the advection-dispersion equation, Water Resour. Res. 29 (1993) 2399-2413.

[58] R.W. Healy, T.F. Russell, Solution of the advection-dispersion equation in two dimensions by a finite-volume Eulerian-Lagrangian localized adjoint method, Adv. Water Res. 21 (1998) 11-26.

[59] G. Hedstrom, Models of difference schemes for $u_{t}+u_{x}=0$ by partial differential equations, Math. Comp. 29 (1975) 969-977.

[60] I. Herrera, R.E. Ewing, M.A. Celia, T.F. Russell, Eulerian-Lagrangian localized adjoint methods: the theoretical framework, Numer. Methods PDEs 9 (1993) 431-458.

[61] T.J.R. Hughes, Multiscale phenomena: Green functions, the Dirichlet-to-Neumann formulation, subgrid scale models, bubbles, and the origins of stabilized methods, Comput. Methods Appl. Mech. Eng. 127 (1995) 387-401.

[62] T.J.R. Hughes, A.N. Brooks, A multidimensional upwind scheme with no crosswind diffusion, in: T.J.R. Hughes (Ed.), Finite Element Methods for Convection Dominated Flows, Vol. 34, ASME, New York, 1979, pp. 19-35.

[63] T.J.R. Hughes, M. Mallet, A new finite element formulation for computational fluid dynamics: III, The general streamline operator for multidimensional advective-diffusive systems, Comput. Methods Appl. Mech. Eng. 58 (1986) 305-328.

[64] G. Jiang, C.-W. Shu, Efficient implementation of weighted ENO schemes, J. Comput. Phys. 126 (1996) 202-228.

[65] C. Johnson, J. Pitkäranta, An analysis of discontinuous Galerkin methods for a scalar hyperbolic equation, Math. Comp. 46 (1986) 1-26.

[66] C. Johnson, A. Szepessy, P. Hansbo, On the convergence of shock-capturing streamline diffusion finite element methods for hyperbolic conservation laws, Math. Comp. 54 (1990) 107-129.

[67] P.D. Lax, Hyperbolic Systems of Conservation Laws and the Mathematical Theory of Shock Waves, SIAM, Philadelphia, PA, 1973.

[68] P.D. Lax, B. Wendroff, Systems of conservation laws, Comm. Pure Appl. Math. 13 (1960) 217-237.

[69] P. Lesaint, P.A. Raviart, On a finite element method for solving the neutron transport equation, in: DeBoor (Ed.), Mathematics Aspects of Finite Elements in Partial Differential Equations, Academic Press, New York, 1974, pp. 89-123.

[70] R.J. LeVeque, Numerical Methods for Conservation Laws, Birkhäuser, Basel, 1992.

[71] X.-D. Liu, S. Osher, T. Chan, Weighted essentially nonoscillatory schemes, J. Comput. Phys. 115 (1994) 200-212.

[72] P.A. Markowich, C. Ringhofer, S. Schmeiser, Semiconductor Equations, Springer, Vienna, 1990.

[73] K.W. Morton, Numerical Solution of Convection-Diffusion Problems, Applied Mathematics and Computation, Vol. 12, Chapman \& Hall, London, 1996.

[74] K.W. Morton, A. Priestley, E. Süli, Stability of the Lagrangian-Galerkin method with nonexact integration, RAIRO $\mathrm{M}^{2} \mathrm{AN} 22$ (1988) 123-151.

[75] H. Nessyahu, E. Tadmor, Non-oscillatory central differencing for hyperbolic conservation laws, J. Comput. Phys. 87 (1990) 408-463.

[76] S.P. Neuman, An Eulerian-Lagrangian numerical scheme for the dispersion-convection equation using conjugate space-time grids, J. Comput. Phys. 41 (1981) 270-294.

[77] S.P. Neuman, Adaptive Eulerian-Lagrangian finite element method for advection-dispersion equation, Int. J. Numer. Methods Eng. 20 (1984) 321-337. 
[78] S. Osherm S. Chakravarthy, High resolution schemes and the entropy condition, SIAM J. Numer. Anal. 21 (1984) 984-995.

[79] T. Peterson, A note on the convergence of the discontinuous Galerkin method for a scalar hyperbolic equation, SIAM J. Numer. Anal. 28 (1991) 133-140.

[80] O. Pironneau, On the transport-diffusion algorithm and its application to the Navier-Stokes equations, Numer. Math. 38 (1982) 309-332.

[81] H.S. Price, R.S. Varga, J.E. Warren, Applications of oscillation matrices to diffusion-convection equations, J. Math. Phys. 45 (1966) 301-311.

[82] J. Pudykiewicz, A. Staniforth, Some properties and comparative performance of the semi-Lagrangian method of robert in the solution of the advection-diffusion equation, Atmosphere-Ocean 22 (1984) 283-308.

[83] P.-A. Raviart, J.-M. Thomas, A mixed finite element method for second order elliptic problems, in: I. Galligani, E. Magenes (Eds.), Mathematical Aspects of Finite Element Methods, Lecture Notes in Mathematics, Vol. 606, Springer, Berlin, 1977, pp. 292-315.

[84] W.H. Reed, T.R. Hill, Triangular mesh methods for the neutron transport equation, Los Alamos Scientific Laboratory Report LA-UR-73-479, 1973.

[85] P.L. Roe, Some contributions to the modeling of discontinuous flows, Lecture Notes in Applied Mathematics, Vol. 22, 1985, pp. 163-193.

[86] H.G. Roos, M. Stynes, L. Tobiska, Numerical Methods for Singular Perturbed Differential Equations, Convection-Diffusion and Flow Problems, Computational Mathematics, Vol. 24, Springer, Berlin, 1996.

[87] T.F. Russell, Eulerian-Lagrangian localized adjoint methods for advection-dominated problems, in: Griffiths, Watson (Eds.), Proceedings of the 13th Dundee Conference on Numerical Analysis, Pitmann Research Notes in Mathematics Series, Vol. 228, Longman Scientific \& Technical, Harlow, United Kingdom, 1990, pp. 206-228.

[88] T.F. Russell, R.V. Trujillo, Eulerian-Lagrangian localized adjoint methods with variable coefficients in multiple dimensions, Computational Methods in Surface Hydrology, Proceedings of the Eighth International Conference on Computational Methods in Water Resources, Venice, Italy, 1990, pp. 357-363.

[89] T.F. Russell, M.F. Wheeler, Finite element and finite difference methods for continuous flows in porous media, in: R.E. Ewing (Ed.), The Mathematics of Reservoir Simulation, SIAM, Philadelphia, PA, 1984, pp. 35-106.

[90] R. Salmon, Lectures on Geophysical Fluid Dynamics, Oxford University Press, New York, 1998.

[91] Y.T. Shih, H.C. Elman, Modified streamline diffusion schemes for convection-diffusion problems, Comput. Methods Appl. Mech. Eng. 174 (1999) 137-151.

[92] C.-W. Shu, Essentially nonoscillatory (ENO) and weighted essentially nonoscillatory (WENO) schemes for hyperbolic conservation laws, in: Quarteroni (Ed.), Advanced Numerical Approximation of Nonlinear Hyperbolic Equations, Lecture Notes in Mathematics, Vol. 1697, Springer, New York, 1997, pp. 325-432.

[93] J. Smoller, Shock Waves and Reaction-Diffusion Equations, 2nd Edition, Fundamental Principles of Mathematical Sciences, Vol. 258, Springer, New York, 1994.

[94] J.C. Strikwerda, Finite Difference Schemes and Partial Differential Equations, Wadsworth and Brooks/Cole, Pacific Grove, CA, 1989.

[95] P.K. Sweby, High resolution schemes using flux limiters for hyperbolic conservation laws, SIAM J. Numer. Anal. 28 (1991) 891-906.

[96] G. Thomaidis, K. Zygourakis, M.F. Wheeler, An explicit finite difference scheme based on the modified method of characteristics for solving diffusion-convection problems in one space dimension, Numer. Methods Partial Differential Equations 4 (1988) 119-138.

[97] L.N. Trefethen, Group velocity in finite difference schemes, SIAM Rev. 24 (1982) 113-136.

[98] B. van Leer, Towards the ultimate conservative difference scheme II, Monotonicity and conservation combined in a second order scheme, J. Comput. Phys. 14 (1974) 361-370.

[99] B. van Leer, On the relation between the upwind-differencing schemes of Godunov, Engquist-Osher, and Roe, SIAM J. Sci. Statist. Comput. 5 (1984) 1-20.

[100] J. Von Neumann, R.D. Richtmyer, A method for the numerical calculation of hydrodynamic shocks, J. Appl. Phys. 21 (1950) 232-237.

[101] H. Wang, Eulerian-Lagrangian localized adjoint methods: analyses, implementations, and applications, Ph.D. Thesis, Department of Mathematics, University of Wyoming, 1992. 
[102] H. Wang, A family of ELLAM schemes for advection-diffusion-reaction equations and their convergence analyses, Numer. Meth. PDEs 14 (1998) 739-780.

[103] H. Wang, An optimal-order error estimate for an ELLAM scheme for two-dimensional linear advection-diffusion equations, SIAM J. Numer. Anal. 37 (2000) 1338-1368.

[104] H. Wang, H.K. Dahle, R.E. Ewing, M.S. Espedal, R.C. Sharpley, S. Man, An ELLAM Scheme for advectiondiffusion equations in two dimensions, SIAM J. Sci. Comput. 20 (1999) 2160-2194.

[105] H. Wang, R.E. Ewing, Optimal-order convergence rates for Eulerian-Lagrangian localized adjoint methods for reactive transport and contamination in groundwater, Numer. Methods PDEs 11 (1995) 1-31.

[106] H. Wang, R.E. Ewing, G. Qin, S.L. Lyons, M. Al-Lawatia, S. Man, A family of Eulerian-Lagrangian localized adjoint methods for multi-dimensional advection-reaction equations, J. Comput. Phys. 152 (1999) 120-163.

[107] H. Wang, R.E. Ewing, T.F. Russell, Eulerian-Lagrangian localized methods for convection-diffusion equations and their convergence analysis, IMA J. Numer. Anal. 15 (1995) 405-459.

[108] H. Wang, D. Liang, R.E. Ewing, S.L. Lyons, G. Qin, An ELLAM-MFEM solution technique for compressible fluid flows in porous media with point sources and sinks, J. Comput. Phys. 159 (2000) 344-376.

[109] H. Wang, R.C. Sharpley, S. Man, An ELLAM scheme for advection-diffusion equations in multi-dimensions, in: Aldama et al. (Eds.) Computational Methods in Water Resources XI, Vol. 2, Computational Mechanics Publications, Southampton, 1996, pp. 99-106.

[110] R. Warming, B.J. Hyett, The modified equation approach to the stability and accuracy analysis of finite difference methods. J. Comput. Phys. 14 (1974) 159-179.

[111] J.J. Westerink, D. Shea, Consider higher degree Petrov-Galerkin methods for the solution of the transient convection-diffusion equation, Int. J. Numer. Methods Eng. 28 (1989) 1077-1101.

[112] G. Whitham, Linear and Nonlinear Waves, Wiley, New York, 1974.

[113] D. Yang, A characteristic-mixed method with dynamic finite element space for convection-dominated diffusion problems, J. Comput. Appl. Math. 43 (1992) 343-353.

[114] S.T. Zalesak, Fully multidimensional flux-corrected transport algorithms for fluids, J. Comput. Phys. 31 (1978) $335-362$.

[115] S.T. Zalesak, A preliminary comparison of modern shock-capturing schemes: linear advection equation, in: R. Vichnevetsky, R.S. Stepleman (Eds.) Advances in Computer Methods for PDEs, Vol. 6, IMACS, Amsterdam, 1987, pp. 15-22. 